\title{
Numerical Study of the Interaction of a Titanium(IV) Isopropoxide/p-Xylene Precursor/Solvent Droplet with Hot Convective Air
}

\author{
P. Narasu*1, M. Nanjaiah ${ }^{2}$, I. Wlokas ${ }^{2}$, E. Gutheil ${ }^{1}$ \\ ${ }^{1}$ Interdisciplinary Center for Scientific Computing, Heidelberg University, Heidelberg, Germany \\ ${ }^{2}$ Institute for Combustion and Gas Dynamics, Department of Fluid Dynamics \\ University of Duisburg-Essen, Duisburg, Germany \\ *Corresponding author email: praveen.narasu@iwr.uni-heidelberg.de
}

\begin{abstract}
The heating and evaporation of precursor solutions in hot oxidizing environments is relevant in flame spray pyrolysis (FSP) to produce metal-oxide powders in the nanosize range. The precursor solution is sprayed into a hot oxidizing environment in which it undergoes heating, evaporation, and combustion which may be followed by nanoparticle synthesis. The present numerical study focuses on single spherically symmetric precursor/solvent droplets of titanium(IV) isopropoxide (TTIP) - Ti $\left(\mathrm{OC}_{3} \mathrm{H}_{7}\right)_{4}$ in $p$-xylene $-\mathrm{C}_{6} \mathrm{H}_{4}\left(\mathrm{CH}_{3}\right)_{2}$ at room temperature in convective hot air at atmospheric pressure. The model includes variable liquid and gas thermophysical properties. The rapid-mixing model and the distillation model for a bi-component droplet are used which do not require the physical resolution of the droplet interior. The dependence of the heating and evaporation characteristics of the single precursor/solvent droplet on parameters including the initial droplet size, the initial TTIP mass fraction in the droplet, the ambient temperature, and the relative gas and droplet velocity is studied. The results may be used in more complex simulations of FSP.
\end{abstract}

\section{Keywords}

bi-component droplet, titanium(IV) isopropoxide, $p$-xylene, heating and evaporation, precursor/solvent droplet

\section{Introduction}

Flame spray pyrolysis (FSP) is a very promising method to produce nanoparticles with special properties. The process involves breakup, atomization, heating, and evaporation of the liquid precursor solution which, after transformation into the gas phase, undergoes combustion and possible succeeding formation of nanoparticles. Therefore, it is important to understand the underlying physical processes, in particular the evaporation process of the single precursor/solvent droplet [1]. In many precursor solutions, the physical properties of the components are not known which imposes a challenge to the problem.

Weise et al. [2] conducted a numerical and experimental investigation of the complex processes involving the synthesis of $\mathrm{TiO}_{2}$ nanoparticles inside a spray flame reactor using TTIP/iso-propyl alcohol as the precursor solution. They found the entire setup of the spray flame reactor particularly suitable to study the influence of the mass flow rate and the precursor/solvent composition on the final particle size. Chang et al. [3] experimentally investigated the synthesis of $\mathrm{TiO}_{2}$ nanoparticles from a TTIP/ethanol precursor solution via FSP. They concluded that a high flame temperature and a low precursor concentration in the solvent increased the droplet evaporation and eventually, the chemical reactions resulted in the formation of smaller nanoparticles.

$\mathrm{Li}$ et al. [4] experimentally examined the micro-explosion mechanism of isolated TTIP/xylene droplets and observed that TTIP does not react with water vapor produced during the combustion process. In experiments by Gonchikzhapov and Kasper [5], the thermal decomposition of a spray consisting of different precursor solutions, for instance iron pentacarbonyl, ferrocene, 
and TTIP in solutions of $o$-xylene or ethanol was studied, and it was found that in solutions of TTIP and o-xylene, there is no chemical interaction between the precursor and the solvent in the liquid phase. Abdelsamie et al. [6] performed direct numerical simulations of a configuration closely related to the SpraySyn burner [7] using a TTIP/ethanol precursor solution to produce $\mathrm{TiO}_{2}$ nanoparticles. The simulations took into account the complete scenario all the way to the production of nanoparticles, including evaporation of the precursor solution and gas phase combustion kinetics. Boichot and Krumdieck [8] modeled the evaporation of TTIP/hexane and TTIP/toluene droplets under vacuum in a pulsed-pressure chemical vapor deposition process. In the zero-dimensional droplet evaporation model, they assumed constant liquid-phase properties of the mixture except for the vapor pressure. They reported that thermodynamics and heat transfer primarily controlled the evaporation kinetics of the precursor/solvent droplets.

The precursor/solvent droplet evaporation plays an important role in FSP. The major difficulty with respect to the droplet evaporation modeling is the typically unknown thermophysical properties of the precursor solution. In the present study, the precursor solution considered is Titanium(IV) Isopropoxide (TTIP) in $p$-xylene, which may be used to produce $\mathrm{TiO}_{2}$ nanoparticles $[9,10,2]$. These nanoparticles are used as photocatalyst and for gas sensors [3]. Due to its non-toxic nature [9], $\mathrm{TiO}_{2}$ is also used as a coating material and in controlled drug delivery applications [11]. The solvent $p$-xylene is chosen mainly because it does not react with TTIP [5].

The following section provides the details concerning the modeling and simulation of the heating and evaporation of TTIP/p-xylene droplets in hot convective air.

\section{Mathematical Model}

A spherically symmetric precursor/solvent droplet is assumed in a convective gas stream at atmospheric pressure. Low droplet Reynolds numbers up to about twenty are considered which ensures that there is neither inner recirculation of the droplet nor flow separation in the wake of the droplet. For the liquid precursor/solvent droplets of TTIP/p-xylene, the droplet heating and evaporation are described using the distillation-limit model and the rapid-mixing model, respectively, for low thermal and mass Peclet numbers [12]. Hence, there is no spatial resolution of the droplet interior and all characteristic variables vary only with time. The governing equations for the convective droplet heating and evaporation are chosen following the study of Abramzon and Sirignano [13] with an extension of Brenn et al. [14] for multicomponent droplets.

In the present model, it is assumed that the droplet is spherical all the times, the solubility of air in the liquid is negligible, mass diffusion due to temperature and pressure gradients is negligible, the gas phase is in a quasi-steady state, the droplet evaporates in a non-reacting inert environment, and heat transfer due to radiation is negligible [14]. The real behavior of the TTIP/p-xylene droplets is considered through use of the non-random two-liquid (NRTL) model [15] with appropriate activity coefficients [16].

\section{Convective Droplet Evaporation}

The droplet evaporation is described by the rapid-mixing model the applicability of which for the present conditions has been discussed by Narasu et al. [12] among others.

Brenn et al. [14] extended the Abramzon and Sirignano [13] model to account for the mass evaporation rate $\dot{m}$ of a multicomponent droplet

$$
\dot{m}=\sum_{i=1}^{n} 2 \pi r_{\mathrm{d}, i} \rho_{\mathrm{f}} D_{\mathrm{f}, i} \widetilde{\operatorname{Sh}} \ln \left(1+B_{\mathrm{M}, i}\right) .
$$

Here, $r_{\mathrm{d}, i}$ denotes the instantaneous droplet radius of component $i, \rho_{\mathrm{f}}$ is the density of the mixture in the film, $D_{\mathrm{f}, i}$ denotes the diffusivity of the component $i$ into air in the film, $\widetilde{\text { Sh }}$ is the modified Sherwood number [13] and $B_{\mathrm{M}, i}$ is the Spalding mass transfer number [14] of the 
liquid component $i$

$$
B_{\mathrm{M}, i}=\frac{Y_{\mathrm{s}, i}-Y_{\infty, i}}{1-Y_{\mathrm{s}, i}}
$$

In Eq. (2), $Y_{\mathrm{s}, i}$ is the mass fraction of the component $i$ at the surface whereas $Y_{\infty, i}$ is the mass fraction of the component $i$ in the ambience. All properties in the film are evaluated using the 1/3-rule [17].

\section{Convective Droplet Heating}

The distillation-limit model [18], extended to account for convection around the droplet [13], is used to describe the droplet heating. Thus, the temperature variation at the droplet surface, $T_{\mathrm{s}}$, yields

$$
m c_{\mathrm{pl}} \frac{\mathrm{d} T_{\mathrm{s}}}{\mathrm{d} t}=2 \pi r_{\mathrm{d}} \lambda_{\mathrm{f}} \widetilde{\mathrm{Nu}} \ln \left(1+B_{\mathrm{T}}\right) \frac{T_{\mathrm{g}}-T_{\mathrm{s}}}{B_{\mathrm{T}}}-\sum_{i=1}^{n} \dot{m}_{i} L_{\mathrm{v}, i}\left(T_{\mathrm{s}}\right) \equiv Q_{1}
$$

where the Spalding heat transfer number, $B_{\mathrm{T}}$ is defined as $[13,14]$

$$
B_{\mathrm{T}}=\left(1+\sum_{i=1}^{n} Y_{\mathrm{i}} B_{\mathrm{M}, i}\right)^{\phi}-1.0 \quad \text { with } \quad \phi=\frac{c_{\mathrm{pl}}}{c_{\mathrm{pf}}} \frac{\widetilde{\mathrm{Nu}}}{\widetilde{\mathrm{Ne}}} .
$$

The parameter $\phi$ accounts for the differences in heat and mass transfer where $c_{\mathrm{pf}}$ and $c_{\mathrm{pl}}$ are the specific heat capacity in the film and of the liquid mixture, respectively, and Le is the Lewis number. Equation (3) represents the heat transfer $Q_{1}$ between the liquid and the gas phase, $m$ is the droplet mass, $\lambda_{\mathrm{f}}$ is the thermal conductivity of the gas mixture in the film, $\mathrm{Nu}$ is the modified Nusselt number [13], $T_{\mathrm{g}}$ is the ambient gas temperature and $L_{\mathrm{v}, i}\left(T_{\mathrm{S}}\right)$ is the temperaturedependent latent heat of vaporization of the component $i$ at the surface temperature $T_{\mathrm{s}}$ of the droplet.

\section{Physical Properties}

Keller et al. [16] measured the thermophysical properties of the solutions of TTIP/p-xylene at atmospheric pressure where solvent compositions range from pure TTIP to pure $p$-xylene. The data include the density, viscosity, thermal conductivity, isobaric specific heat capacity, and vapor-liquid equilibrium. The experimental relative standard uncertainty ranges from $0.04 \%$ for the density to $3 \%$ for the mixture viscosity. From the experimental thermophysical data, empirical correlations [16] for these properties were established which are used in the present study. These thermophysical properties are valid within the temperature range of $293.15 \mathrm{~K}$ to $373.15 \mathrm{~K}$, beyond which the properties are extrapolated. The vapor pressure of both TTIP and $p$-xylene is calculated using the Antoine equation for which the parameters are provided by Keller et al. [16]. It is expected that the liquid mixture may not be ideal, and therefore, real behavior is considered through the use of activity coefficients of TTIP and $p$-xylene which

Table 1. Physical properties of TTIP and p-xylene at $293.15 \mathrm{~K}$ and $1 \mathrm{~atm}$

\begin{tabular}{l|r|r}
\hline & TTIP $-\mathrm{Ti}\left(\mathrm{OC}_{3} \mathrm{H}_{7}\right)_{4}$ & $p$-xylene $-\mathrm{C}_{6} \mathrm{H}_{4}\left(\mathrm{CH}_{3}\right)_{2}$ \\
\hline Boiling point [K] & $505.00[19]$ & $411.50[20]$ \\
Molar mass [g/mol] & $284.22[19]$ & $106.16[21]$ \\
Density $\left[\mathrm{kg} / \mathrm{m}^{3}\right]$ & $966.00[16]$ & $860.90[16]$ \\
Specific heat capacity [J/(mol K)] & $457.60[16]$ & $182.60[16]$ \\
Latent heat of vaporization $[\mathrm{J} / \mathrm{mol}]$ & $64,029.08[22]$ & $42,675.25[20]$ \\
\hline
\end{tabular}


are modeled using the non-random two-liquid (NRTL) method [15] by calculating the activity coefficients using the vapor-liquid equilibrium data from Keller et al. [16].

Variable gas properties of TTIP are computed from the work of Buerger et al. [23] and Shmakov et al. [24]. The mixture properties of TTIP and p-xylene in the gas phase are taken from the study of Poling and Prausnitz [22]. In particular, the thermal conductivity of the gas mixture is calculated using the Wassiljewa equation. Wilke's rule is employed to evaluate the viscosity of the gas mixture in the film. The diffusivity of TTIP vapor and $p$-xylene vapor in air is evaluated using the Fuller equation. The temperature dependence of the latent heat of evaporation of TTIP is described using the Watson relation [22] and that of $p$-xylene is taken from the book of Majer and Svoboda [20].

Table 1 provides physical properties of TTIP and $p$-xylene at NTP which shows that $p$-xylene is the component with higher volatility compared to TTIP.

\section{Results and Discussion}

The present study focuses on the heating and evaporation characteristics of a single droplet consisting of a precursor solution of TTIP and $p$-xylene. Unfortunately, there is no experimental data for comparison with the numerical results. However, the bi-component droplet heating and evaporation model was validated for single bi-component ethanol/water droplets in air [12] with experimental results from $\mathrm{Ma}$ et al. [25] so that for the present system under consideration, numerical results of the physical process as well as a parameter study are presented.

Following the conditions in experimental studies of FSP $[7,5,4]$, initial droplet radii of $5 \mu \mathrm{m}$, $10 \mu \mathrm{m}, 25 \mu \mathrm{m}$, and $50 \mu \mathrm{m}$ are considered for ambient air temperatures of $800 \mathrm{~K}$ and $1200 \mathrm{~K}$ and relative velocities of $4 \mathrm{~m} / \mathrm{s}$ and $8 \mathrm{~m} / \mathrm{s}$. Also, three different initial mass fractions of TTIP in the precursor solution are considered, namely, 0.025, 0.155 and 0.25 . For these conditions, the Weber numbers stay well below the critical Weber number so that droplet breakup does not occur. Also, both thermal and mass Peclet numbers are in a range in which the distillation-limit and the rapid-mixing models are valid [12].

Figure 1 shows the temporal variation of the normalized droplet surface area for TTIP/p-xylene droplet of initial droplet radius $5 \mu \mathrm{m}$ (left) and $25 \mu \mathrm{m}$ (right) at a relative velocity of $4 \mathrm{~m} / \mathrm{s}$ for different initial TTIP mass fractions of $0.0,0.025,0.155$ and 0.25 in the precursor droplets. Ambient gas temperatures of $800 \mathrm{~K}$ (broken lines) and $1200 \mathrm{~K}$ (solid lines) are considered.

In all cases, thermal expansion of the droplet occurs as seen in Fig. 1, where the normalized droplet surface area increases initially due to the initial droplet heating. The droplet expansion reflects the variable physical properties of the liquid, in particular that of the liquid density. At an ambient temperature of $1200 \mathrm{~K}$ the droplet expands approximately $4.5 \%$ of the initial droplet surface area, which is about one percent higher than the droplet expansion observed at $800 \mathrm{~K}$, reflecting the stronger variation of liquid density with temperature. The unsteady droplet
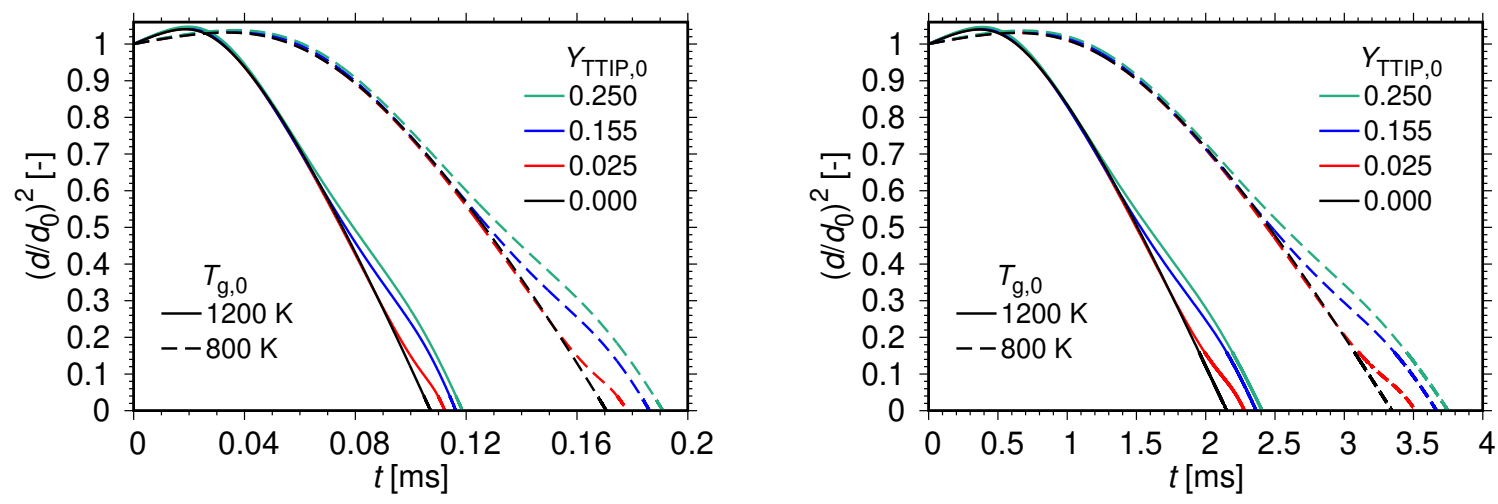

Figure 1. Normalized droplet surface area with time. Initial conditions: $T_{1,0}=293.15 \mathrm{~K}, p=1 \mathrm{bar}, u_{0}=4 \mathrm{~m} / \mathrm{s}$. Left: $r_{\mathrm{d}, 0}=5 \mu \mathrm{m}$. Right: $r_{\mathrm{d}, 0}=25 \mu \mathrm{m}$. 

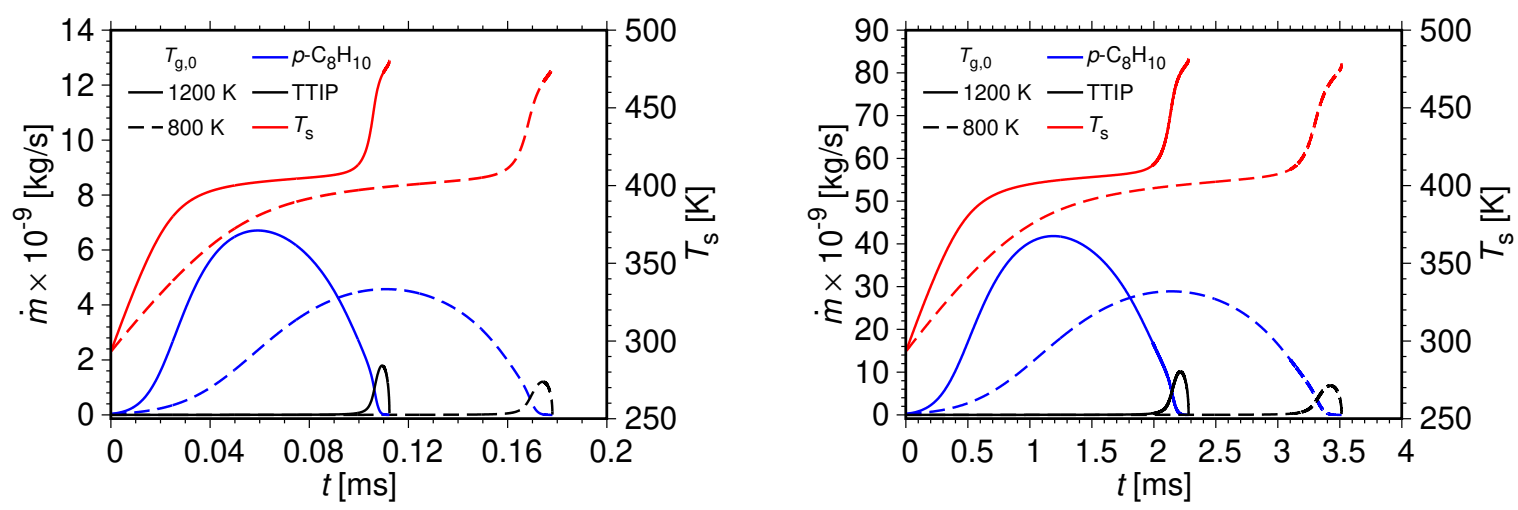

Figure 2. Mass evaporation rate with time. Initial conditions: $T_{1,0}=293.15 \mathrm{~K}, p=1$ bar, $u_{0}=4 \mathrm{~m} / \mathrm{s}$, $Y_{p-\mathrm{C}_{8} \mathrm{H}_{10}, 0}=0.975, Y_{\mathrm{TTIP}, 0}=0.025$. Left: $r_{\mathrm{d}, 0}=5 \mu \mathrm{m}$ Right: $r_{\mathrm{d}, 0}=25 \mu \mathrm{m}$.
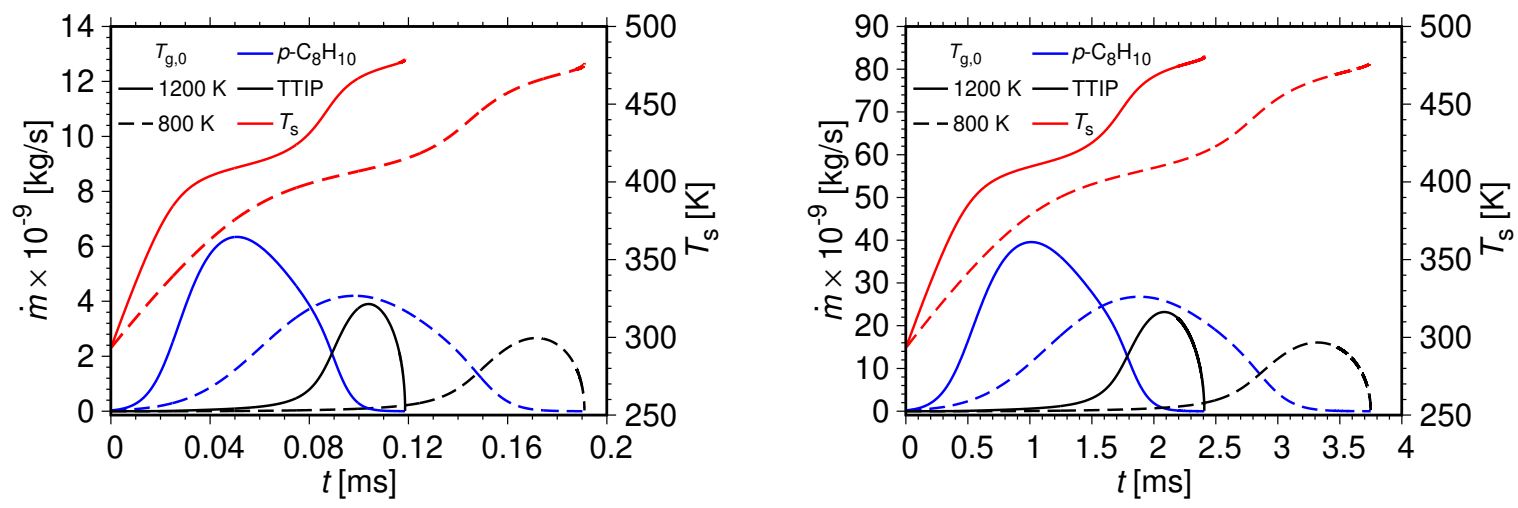

Figure 3. Mass evaporation rate with time. Initial conditions: $T_{1,0}=293.15 \mathrm{~K}, p=1$ bar, $u_{0}=4 \mathrm{~m} / \mathrm{s}$, $Y_{p-\mathrm{C}_{8} \mathrm{H}_{10}, 0}=0.75, Y_{\mathrm{TTIP}, 0}=0.25$. Left: $r_{\mathrm{d}, 0}=5 \mu \mathrm{m}$ Right: $r_{\mathrm{d}, 0}=25 \mu \mathrm{m}$.

heating is followed by quasi-steady droplet evaporation, where preferential evaporation of $p$ xylene occurs due its higher volatility, cf. Tab. 1, followed by the evaporation of TTIP. Due to increased heat transfer between the droplet and the ambience, the droplet evaporates faster at the higher ambient temperature, which results in a reduced droplet lifetime of almost $30 \%$ at $1200 \mathrm{~K}$ compared to $800 \mathrm{~K}$. For the initial droplet radius of $25 \mu \mathrm{m}$, cf. Fig. 1 (right), the droplet lifetime increases due to the larger mass of the droplet that is to be evaporated. For fixed ambient temperature, an increase in initial mass fraction of TTIP in the droplet prolongs the droplet evaporation process due to the lower volatility of TTIP compared to $p$-xylene. Towards the end of the quasi-steady evaporation period, there is some deviation of linearity of $\left(d / d_{0}\right)^{2}$ with time, which is analyzed in Figs. 2 and 3.

The mass evaporation rates of the two droplet components as well as the droplet surface temperatures for the lowest and the highest initial TTIP mass fraction in the droplets are shown in Figs. 2 and 3, respectively, where the left parts show the results for an initial droplet radius of $5 \mu \mathrm{m}$ and the right parts those for $25 \mu \mathrm{m}$ for a relative velocity of $4 \mathrm{~m} / \mathrm{s}$. Solid lines show results for an ambient temperature of $1200 \mathrm{~K}$ and broken lines those for $800 \mathrm{~K}$.

After an initial increase of droplet surface temperature which is associated with droplet expansion, Figs. 2 and 3 show a dominant evaporation of the higher volatile $p$-xylene. TTIP shows enhanced evaporation after a second increase in droplet surface temperature which reflects the lower volatility as well as the higher boiling temperature, see Tab. 1. These characteristics prevail for all conditions under consideration. The larger initial droplet size leads to higher mass evaporations, please note the different scales in the left and right plots in Figs. 2 and 3. Also, the mass evaporation of TTIP is larger for the higher initial mass fraction of TTIP in the droplets as expected. A comparison of the left and right parts of the figures reveals that normalized profiles with respect to droplet lifetime and maximum mass evaporation for both droplet sizes 

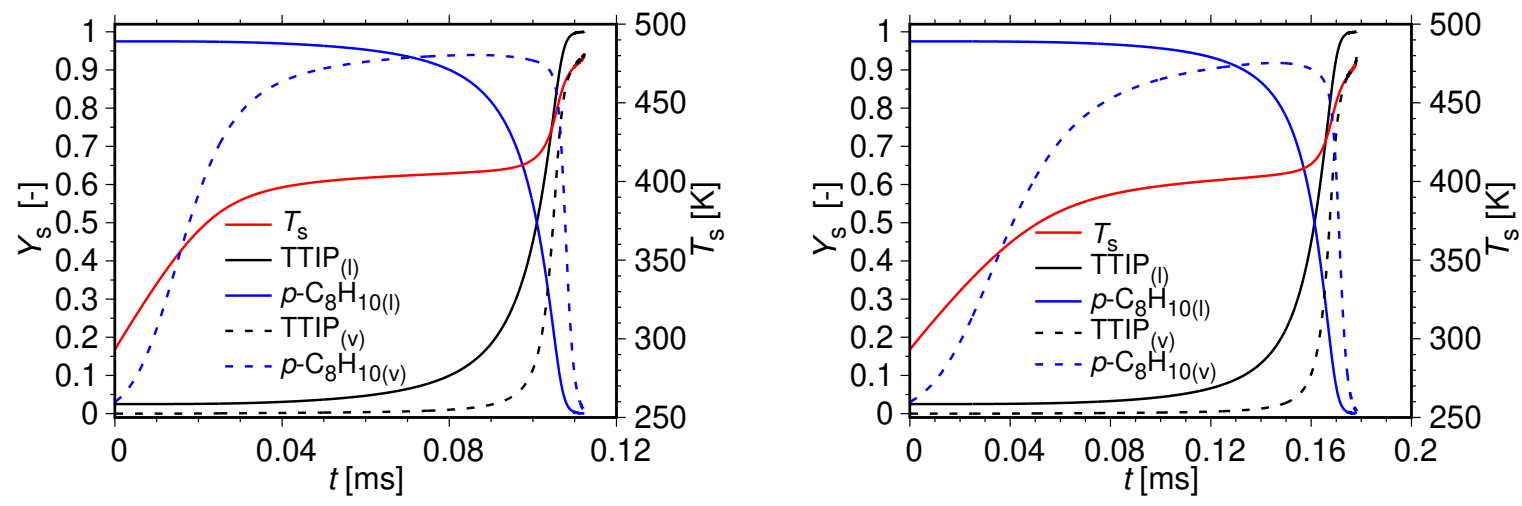

Figure 4. Mass fractions of species and gas temperature at the droplet surface. Initial conditions: $r_{\mathrm{d}, 0}=5 \mu \mathrm{m}$, $T_{1,0}=293.15 \mathrm{~K}, p=1$ bar, $u_{0}=4 \mathrm{~m} / \mathrm{s}, Y_{p-\mathrm{C}_{8} \mathrm{H}_{10}, 0}=0.975, Y_{\mathrm{TTIP}, 0}=0.025$. Left: $T_{\mathrm{g}, 0}=1200 \mathrm{~K}$. Right: $T_{\mathrm{g}, 0}=800 \mathrm{~K}$.

shows qualitatively the same characteristics as was also found for the heating, evaporation, and thermal decomposition of INN/ethanol droplets under similar conditions [26].

Figure 4 exemplarily shows the profiles of temperature and mass fractions of the species at the droplet surface for the conditions of left side of Fig. 2 for an ambient temperature of $1200 \mathrm{~K}$, cf. Fig. 4 (left) and $800 \mathrm{~K}$ (right). Besides the droplet surface temperature, the mass fractions of TTIP and $p$-xylene are displayed in both gas and liquid phase. The profiles of the mass fractions reflect the processes associated with the mass evaporation rates of the different species and that of the droplet surface temperature.

The final decision on the choice of conditions for an FSP will depend on the requirements of the global process and the conditions under which the desired properties of the final nanoparticles are to be formed. The choice of parameters such as initial droplet sizes of the precursor solution will also depend on the droplet environment such as relative droplet velocity and ambient gas temperature. The droplet lifetimes will play a major role in evaluating the total process times, and therefore, the duration of the process is studied next.
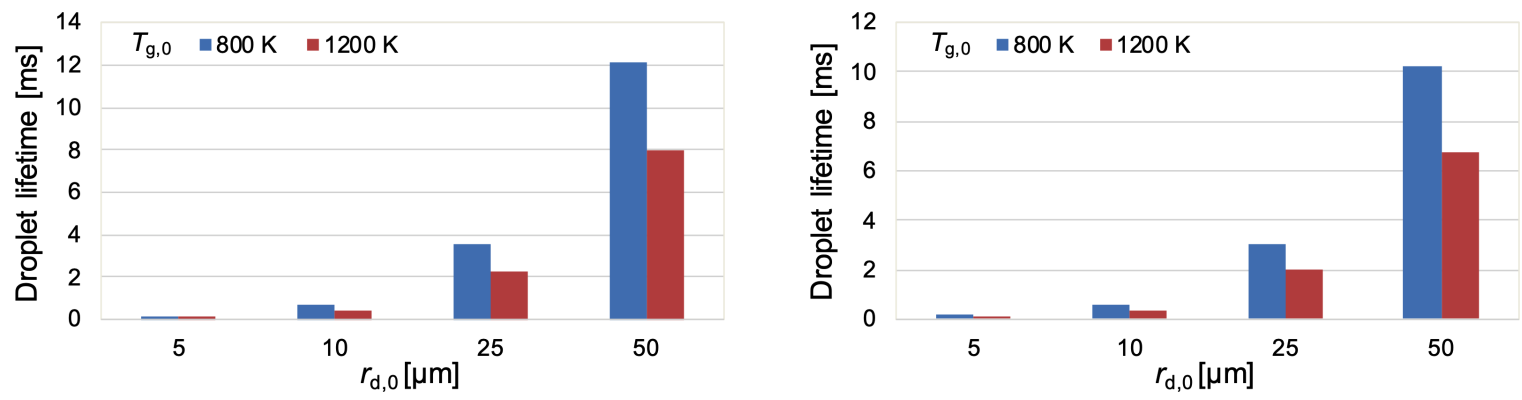

Figure 5. Droplet lifetime for different initial droplet sizes and ambient gas temperatures. $T_{1,0}=293.15 \mathrm{~K}, p=1 \mathrm{bar}$, $Y_{p-\mathrm{C}_{8} \mathrm{H}_{10}, 0}=0.975, Y_{\mathrm{TTIP}, 0}=0.025$. Left: $u_{0}=4 \mathrm{~m} / \mathrm{s}$. Right: $u_{0}=8 \mathrm{~m} / \mathrm{s}$.
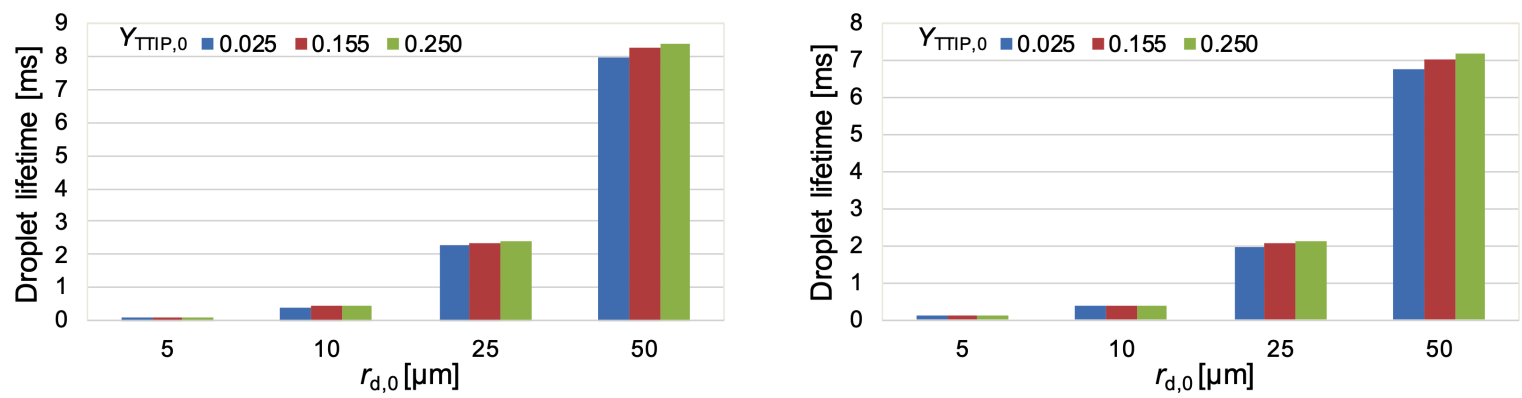

Figure 6. Droplet lifetime for different initial droplet sizes and different precursor/solvent compositions. $T_{1,0}=$ $293.15 \mathrm{~K}, p=1$ bar, $T_{\mathrm{g}, 0}=1200 \mathrm{~K}$. Left: $u_{0}=4 \mathrm{~m} / \mathrm{s}$. Right: $u_{0}=8 \mathrm{~m} / \mathrm{s}$. 
Figures 5 and 6 display the droplet lifetimes for different initial droplet radii of $5 \mu \mathrm{m}, 10 \mu \mathrm{m}$, $25 \mu \mathrm{m}$, and $50 \mu \mathrm{m}$ for different ambient gas temperatures and different precursor/solvent compositions, respectively. The left parts of these figures show the lifetimes for an initial relative velocity of $4 \mathrm{~m} / \mathrm{s}$ and the right part those for $8 \mathrm{~m} / \mathrm{s}$.

The increase in relative velocity from $4 \mathrm{~m} / \mathrm{s}$ to $8 \mathrm{~m} / \mathrm{s}$ enhances droplet evaporation [18, 13], resulting in some reduction in droplet lifetimes. This is typical for droplet evaporation in dry air [12]. At both ambient temperatures, the droplet lifetimes increase with droplet size due to prolonged droplet heating and evaporation times of the larger droplet. The droplet lifetimes are reduced at higher ambient temperatures due to the increased heat transfer rates. Increasing the initial TTIP mass fraction in the precursor solution droplet results in the droplet eventually taking more time to evaporate due to the lower volatility of TTIP compared to $p$-xylene and the higher boiling temperature of TTIP.

The parametric study of the heating and vaporization characteristics of TTIP/p-xylene precursor solution droplets may be used in more complex simulations of FSP.

\section{Summary and Conclusions}

A numerical study of the heating and evaporation of single TTIP/p-xylene precursor/solvent droplets in hot convective air has been performed for use in the modeling of flame spray pyrolysis (FSP) to produce $\mathrm{TiO}_{2}$ nanoparticles. The model includes the heating and evaporation of a bi-component droplet where new physical properties [16] of TTIP and $p$-xylene were used. The single precursor solution droplet initially shows some expansion due to droplet heating, reflecting the variable liquid properties which are used in the simulation. The first period of the droplet evaporation is dominated by the evaporation of the higher volatile component $p$-xylene and latter, TTIP with its high boiling temperature vaporizes, generating a gaseous mixture of TTIP and $p$-xylene that may chemically react in an hot oxidizing environment, which is not part of the present study.

Parametric droplet lifetimes depending on the ambient gas temperature, the relative gas and droplet velocity, the initial droplet size as well as the initial mass fraction of TTIP in the precursor solution droplet are determined.

The present results of the TTIP/p-xylene droplets may be used in more complex simulations of flame spray pyrolysis.

\section{Acknowledgements}

Funding by the Deutsche Forschungsgemeinschaft (DFG, German Research Foundation) Projektnummer 374463455 is gratefully acknowledged.

\section{References}

[1] Kammler, H.K., Mädler, L., Pratsinis, S.E., 2001. Chemical Engineering \& Technology: Industrial Chemistry-Plant Equipment-Process Engineering-Biotechnology 24, 583-596.

[2] Weise, C., Menser, J., Kaiser, S., Kempf, A., Wlokas, I., 2015. Proceedings of the Combustion Institute 35, 2259-2266.

[3] Chang, H., Kim, S.J., Jang, H.D., Choi, J.W., 2008. Colloids and Surfaces A: Physicochemical and Engineering Aspects 313, 282-287.

[4] Li, H., Rosebrock, C., Riefler, N., Wriedt, T., Mädler, L., 2017. Proceedings of the Combustion Institute 36, 1011-1018.

[5] Gonchikzhapov, M., Kasper, T., 2020. Industrial \& Engineering Chemistry Research 59, 8551-8561. 
[6] Abdelsamie, A., Kruis, F.E., Wiggers, H., Thévenin, D., 2020. Flow, Turbulence and Combustion 105, 497-516.

[7] Schneider, F., Suleiman, S., Menser, J., Borukhovich, E., Wlokas, I., Kempf, A., Wiggers, H., Schulz, C., 2019. Review of Scientific Instruments 90, 085108.

[8] Boichot, R., Krumdieck, S., 2015. Chemical Vapor Deposition 21, 375-384.

[9] Teisala, H., Tuominen, M., Aromaa, M., Mäkelä, J., Stepien, M., Saarinen, J., Toivakka, M., Kuusipalo, J., 2010. Surface and Coatings Technology 205, 436-445.

[10] Wang, W.N., Lenggoro, I.W., Terashi, Y., Kim, T.O., Okuyama, K., 2005. Materials Science and Engineering: B 123, 194-202.

[11] Dong, H., Tang, G., Ma, T., Cao, X., 2016. Journal of Materials Science: Materials in Medicine 27, 1-8.

[12] Narasu, P., Boschmann, S., Pöschko, P., Zhao, F., Gutheil, E., 2020. Combustion Science and Technology 192, 1233-1252.

[13] Abramzon, B., Sirignano, W., 1989. International Journal of Heat and Mass Transfer 32, 1605-1618.

[14] Brenn, G., Deviprasath, L., Durst, F., Fink, C., 2007. International Journal of Heat and Mass Transfer 50, 5073-5086.

[15] Renon, H., Prausnitz, J.M., 1968. AIChE journal 14, 135-144.

[16] Keller, A., Wlokas, I., Kohns, M., Hasse, H., 2020. Journal of Chemical \& Engineering Data 65, 869-876.

[17] Hubbard, G., Denny, V., Mills, A., 1975. International Journal of Heat and Mass Transfer 18, 1003-1008.

[18] Law, C.K., 1982. Progress in Energy and Combustion Science 8, 171-201.

[19] Titanium isopropoxide - Wikipedia, The Free Encyclopedia. https://en.wikipedia.org/ w/index.php?title=Titanium_isopropoxide\&oldid=1005040488.

[20] Majer, V., Svoboda, V., 1985. Enthalpies of Vaporization of Organic Compounds: A Critical Review and Data Compilation. Blackwell Scientific Publications, Oxford.

[21] Encyclopedia, P.X.W.T.F. https://en.wikipedia.org/w/index.php?title=P-Xylene\& oldid=1007917984.

[22] Poling, B.E., Prausnitz, O'Connell, J.P., 2001. The properties of gases and liquids. volume 5. McGraw-Hill New York.

[23] Buerger, P., Nurkowski, D., Akroyd, J., Kraft, M., 2017. Proceedings of the Combustion Institute 36, 1019-1027.

[24] Shmakov, A., Korobeinichev, O., Knyazkov, D., Paletsky, A., Gerasimov, I., Bolshova, T., Kiselev, V., Gritsan, N., 2013. Proceedings of the Combustion Institute 34, 1143-1149.

[25] Ma, L., Chou, X., Cui, Y., Zheng, Z., 2014. Industrial Heating 43, 13-16.

[26] Narasu, P., Keller, A., Kohns, M., Hasse, H., Gutheil, E., 2020 submitted. International Journal of Thermal Sciences. 